\title{
Paraspinal Muscle Activity during Unstable Superman and Bodyweight Squat Exercises
}

\author{
Fernando Carvalheiro Reiser ${ }^{1, *}$, Bruno Gonçalves Durante ${ }^{1,2}$, William Cordeiro de Souza ${ }^{3}$ \\ Luis Paulo Gomes Mascarenhas ${ }^{3}$ and Giordano Márcio Gatinho Bonuzzi ${ }^{4,5}$ \\ 1 Laboratory of Sciences in Physical Activity, School of Arts, Sciences and Humanities, \\ University of São Paulo, 03828-000 São Paulo, Brazil; bruno_crvg@hotmail.com \\ 2 Health Sciences Center; Department of Physical Education, Laboratory of Exercise Physiology \\ and Kinesiology, University of Vale do Itajaí, 88302-901 Itajaí, Brazil \\ 3 Department of Physical Education, University of Contestado, 89460-000 Canoinhas, Brazil; \\ professor_williamsouza@yahoo.com.br (W.C.d.S.); masca58@hotmail.com (L.P.G.M.) \\ 4 Laboratory of Motor Behavior, School of Physical Education and Sport, University of São Paulo, \\ 05508-030 São Paulo, Brazil; giordanomgb@gmail.com \\ 5 Department of Physical Education, Paulista University-UNIP, 01533-000 São Paulo, Brazil \\ * Correspondence: fcreiser@gmail.com; Tel.: +55-47-9955-0273
}

Academic Editor: Giuseppe Musumeci

Received: 2 December 2016; Accepted: 6 February 2017; Published: 27 February 2017

\begin{abstract}
The purpose of this study was to investigate the recruitment of the paraspinal muscles longissimus and iliocostalis during dynamic contractions of superman exercises on stable and unstable surfaces, and during unloaded bodyweight squats. Surface electromyography (EMG) was measured from these two muscles bilaterally while participants performed the exercises. Participants ( $n=17$, age $24 \pm 3.1$ years, body mass $78.5 \pm 8.2 \mathrm{~kg}$, height $1.81 \pm 7.4 \mathrm{~m})$ performed two sets of 10 repetitions for each exercise in a random order. Correlation analysis demonstrated a strong positive relation between both sides of the participants (longissimus pairs: $r=0.945$; and iliocostalis pairs: $r=0.855)$, demonstrating a symmetric muscle recruitment in all exercises. However, there were no significant differences between the exercises for both muscles in a multiple comparison analysis (longissimus pairs: $p=0.496$; and iliocostalis pairs: $p=0.296$ ). The bodyweight squat produces similar muscle activity of the longissimus and iliocostalis compared with superman exercises on stable and unstable surfaces. Considering the dynamic nature of the bodyweight squat, and its implications on functional daily living activities and sports conditions, it seems a more reasonable choice for targeting paraspinal muscles if compared to superman exercises or unstable superman exercises.
\end{abstract}

Keywords: squat; lumbar hyperextension; resistance training; low back pain; posterior trunk electromyography

\section{Introduction}

Core muscles are composed essentially of the muscles of the lumbar spine and abdominal wall along with the back extensors and the quadratus lumborum. Together they provide stability to the torso and control in all three planes of motion for multidirectional activities of sports and daily living [1-3]. Individuals with acute or chronic low back pain demonstrate decreased muscle strength and endurance in abdominal wall muscles and back extensors, which reduces the ability to restrict compressive forces and shear in the vertebral spine [4-6].

Because of this, stability and endurance enhancement of the paraspinal muscles are essential for the prevention and/or treatment of low back disorders, as they increase the muscles' capacity to restrict external forces on the soft tissues of the spinal column $[5,6]$. The literature provides good prognostic 
findings about such a stabilization training intervention after a 10-week program, including augmentation of the muscle cross-sectional area and a 50.1\% reduction of low back pain, as measured via the visual analog scale. Such results clearly improve the quality of life of individuals with low back pain [7].

Therefore, rehabilitation programs for low back disorders incorporate several exercises that target paraspinal muscles. Prone-lying trunk-extension activities such as the superman exercise induce higher activation of the paraspinal muscles, superficial fibers (longissimus and iliocostalis), and deep fibers (multifidus) compared to traditional stabilization drills such as bird dog, abdominal bridge, pelvic thrust and side bridge exercises [3,8-10]. In addition, given that an unstable surface offers different postural control strategies, thereby challenging the entire core complex and augmenting muscle activation, superman exercises performed on a stability ball (unstable superman) have been found to possibly provide a progression of external load. In fact, unstable superman exercises increase the upper lumbar and lumbosacral spinae erectors (as seen in electromyography recordings) compared with the stable version [8].

Nonetheless, Ekstrom et al.'s [9] study demonstrated that the muscular recruitment derived from stable superman exercises is 30\% higher than that of the adapted unstable Superman exercises, particularly regarding longissimus thoracis and lumbar multifidus recruitment. These contradictory results regarding unstable and core muscle recruitment may be related to body position in the function of the instability apparatus because, depending on the center of mass position on the instability apparatus, it is possible to provide more stable or unstable posture requirements, thus influencing muscle recruitment [11].

However, one of the major concerns regarding superman exercises is its hyperextension trunk posture, which may be not tolerable for people with low back disorders $[2,10]$. In this sense, previous studies investigating alternative exercises such as the barbell back squat and deadlift have reported a substantial increase of paraspinal muscles as evidenced by electromyography (EMG) with an $80 \%$ load of maximum repetition compared with superman exercises [12]. Nuzzo et al. [13] also observed the superiority of the barbell back squat and deadlift over the unstable superman exercise. They found that an additional external load increases paraspinal muscle recruitment. Comfort et al. [14] observed no differences for paraspinal muscle activity between front barbell squats and unstable superman exercises. However, there are also findings in the literature that showed more favorable results for unstable superman exercises than for squats and deadlifts with progressive or fixed loads [12-14].

Considering that people with low back disorders may not tolerate performing squats with higher loads, bodyweight squats promote more functional neuromotor recruitment $[2,6,15]$, and there are no kinematic changes from using a barbell in lumbar and sacrum patterns versus the bodyweight squat version [15]. The aim of this study was to verify longissimus and iliocostalis muscle activity during superman exercises in unstable and stable conditions versus their activity in bodyweight squats. Our hypothesis was that we would find symmetrical relations of muscular recruitment, and that the unstable superman exercise would increase the trunk muscle activity the most; superman exercises on stable surfaces would rank second, and bodyweight squats would increase the trunk muscle activity the least of the three.

\section{Materials and Methods}

\subsection{Participants}

Based on a pilot study was determined with software G*Power 3.1 ${ }^{\circledR}$ (Düsseldorf University, Düsseldorf, Germany) which 13 participants would be necessary to an $\alpha$ level of 0.05 , and a power $(1-\beta$ of 0.80$)$ [16], and to allow for potential drop-out, we included 17 physically active men (age $24 \pm 3.1$ years, body mass $78.5 \pm 8.2 \mathrm{~kg}$, height $1.81 \pm 7.4 \mathrm{~m}$ ). Eligibility criteria were: 1-male participants from 20 to 30 years old, 2-at least three years of experience with strength training, 3 -no previous lower back injury in the last twelve months. The experimental procedure was approved by the University of Itajai ethics board's commission (Protocol Number: 135.521; approved: 2 December 2014). 


\subsection{Procedures}

After all participants signed a consent form, they performed all three exercises (unstable Superman exercise, Superman exercise and bodyweight squat) in random order in two separate sessions with a two-day interval. The first session was for familiarization, the EMG recording occurred during the second session. Participants performed two sets of 10 repetitions of each exercise. Participants enjoyed $2 \mathrm{~min}$ rest between sets and $5 \mathrm{~min}$ rest between exercises to avoid fatigue. No external load was used in exercises, because additional load increases significant muscle activity, as shown in previous studies utilizing progressive or fixed loads [12-14]. All participants completed the required number of repetitions of each exercise within the required timespan, with a two-second contraction time (one second for each descend/ascend phase); a metronome was used to normalize time under tension for all participants.

\subsection{Exercise Procedures}

Bodyweight squat: Participants began in a standing position with their hands in front of their chests, with a stance width of $100 \%$ of greater trochanter distance and feet slightly rotated outward. Participants were instructed to maintain a neutral spine curvature during all squat movement avoiding changes in muscle fiber angle [5,15]. Participants performed a knee flexion with the thighs parallel to the floor (knee flexion of approximately 1.70 radians), and then they performed the ascend phase, returning to a standing position while maintaining isometric contraction of the trunk muscles (Figure 1a).

Superman: This activity is a hyperextension exercise in which participants start in a lying-prone position with neutral spine curvatures. Feet were in contact with the floor, legs fully extended, arms flexed and hands placed at the level of the temporal bone for the entire exercise period. Participants were instructed to perform lumbar hypertension (approximately 20 degrees) in the ascend phase and return to a neutral position ( 0 degrees) in the descend phase (Figure $1 b$ ).

Unstable Superman: This entails the same procedures as the Superman exercise; however, the range of motion is adopted in unstable conditions: A Swiss Ball was positioned under the umbilicus region of the participant, ensuring that the feet and legs were fully extended. With arms flexed, hands were placed at the temporal bone level throughout the exercise (Figure 1c).
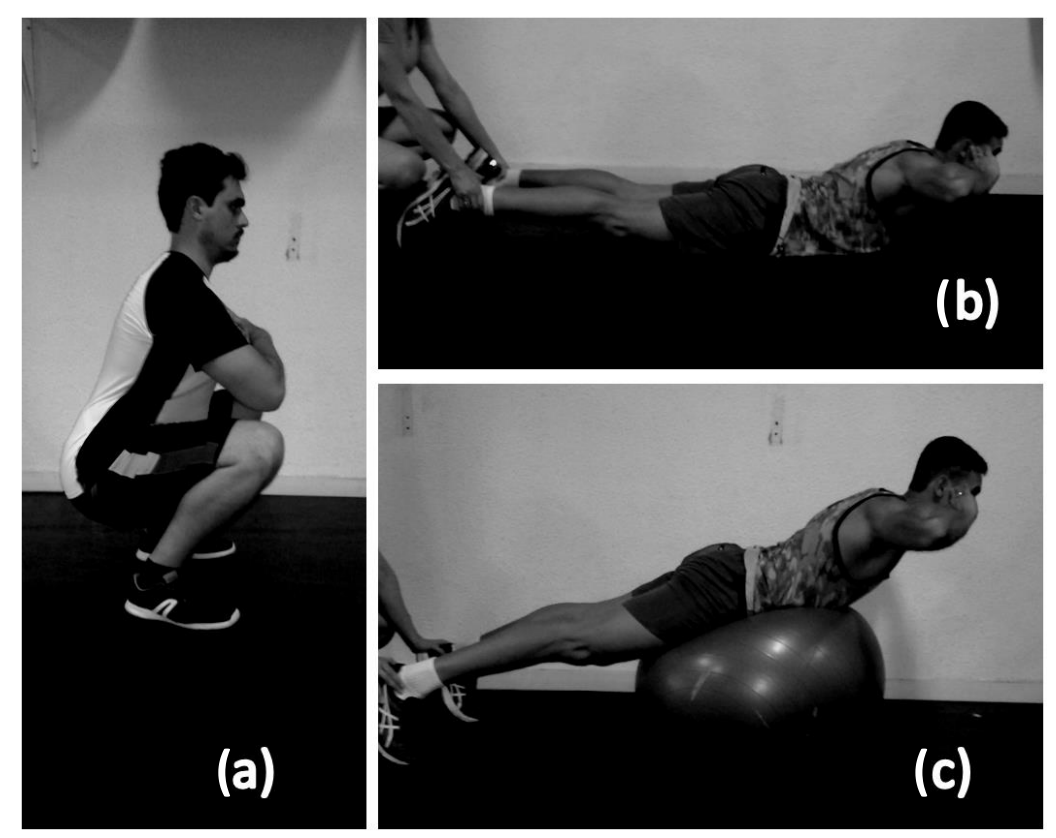

Figure 1. Bodyweight squat exercise (a); superman exercise (b); unstable superman exercise (c). 


\subsection{Electrodes Placement}

All electrodes were placed on both sides of participants' trunk muscles. Two electrodes $\mathrm{Ag} / \mathrm{AgCl}$ with a $20 \mathrm{~mm}$ inter-electrode distance (Miotrace ${ }^{\circledR} 100$ ) were placed at the midline of the trunk muscles parallel to the left longissimus (LL), right longissimus (RL), left iliocostalis (LI), and right iliocostalis (RI) fibers with a reference electrode placed on the right clavicle. Following surface EMG for non-invasive assessment of muscles (SENIAM) recommendations, all electrodes were positioned on participants' skin by the same researcher [17]. Electrode placement is demonstrated in Table 1.

Table 1. Position of electrodes.

\begin{tabular}{cl}
\hline Muscle & \multicolumn{1}{c}{ Electrode Placement } \\
\hline Longissimus & Two finger width lateral from the spine processes of lumbar first vertebra \\
\hline Iliocostalis & $\begin{array}{l}\text { One finger width medial from the line from the posterior spina iliaca superior to the lowest } \\
\text { point of the lower rib, at the level of lumbar second vertebra }\end{array}$ \\
\hline
\end{tabular}

Before the attachment of the electrode, the skin of the participant was prepared by shaving and cleaning with soap. Muscle activity, during the ascent and descent phase, was recorded via Miotool $400^{\circledR}$ (MIOTEC Biomedical Equipament, Porto Alegre/Brazil). The electromyography data of all exercises were collect in the entire cycle of movement from the two sets, but only windows of $0.2 \mathrm{~s}$ from second to octave repetition of the sets were analyzed in order to avoid postural adjustments and provide best quality of movement [18]. Electromyography was analyzed using Miography software package ${ }^{\circledR}$. The distinct dynamic characteristics of all exercises, the normalization method used on Raw EMG signals were based individually in the peak of muscle activation achieve during both sets of each exercise from the Root Mean Square value. The gain was of 1000 times, using a $4^{\circ}$ order Butterworth filter, a pass band of the EMG amplifier of 10-450 Hz, sampling rate of $2000 \mathrm{~Hz}, 6 \mathrm{kOhms}$ of maximum intra-electrode impedance, and common-mode rejection ratio of $100 \mathrm{~dB}$. All sEMG data were notch filtered at frequencies of 59 and $61 \mathrm{~Hz}$ for eliminating any electrical interference and harmonics. Median Frequency (MF) analysis with Fast Fourier Transform (FFT) were made using Miography software package ${ }^{\circledR}$ to verify possible fatigue events, a difference of $15 \%$ from the first to final set of protocol was considered an exclusion criteria, however all participants demonstrated lower values of $\operatorname{MF}(7.32 \% \pm 3.1 \%)$, indicating that rest intervals provide enough time to avoid fatigue events $[19,20]$.

\subsection{Statistical Analysis}

All results from both sets of each exercise are presented as mean and standard deviations (SD). Cohen's d effect size is also presented: an effect size can be considered small (0.2), medium (0.5) and large (0.8). Pearson product-moment correlation coefficient was used to determine if there was a symmetrical relation of muscle sides; a stronger correlation $(>0.7)$ indicated similar muscle-activity intensity from both sides. Posteriorly, a one-way analysis of variance (ANOVA) with repeated measures was used to assess differences in the activity for each muscle measured during the three exercises. Tukey HSD post hoc correction was applied when there were significant statistical differences. Statistical analyses were performed with SPSS version 20.0 (SPSS, Inc., Chicago, IL, USA). Statistical significance was accepted at $p \leq 0.05$.

\section{Results}

There were strong positive correlation coefficients between the sides and exercises for RL and LL $(r=0.945$; and $p=0.065)$ and RI and LI $(r=0.855$; and $p=0.095)$, indicating a relation in which all exercises promoted the recruitment of these muscles in a symmetrical fashion. For the longissimus muscle (Figure 2a), no significant differences were found between exercises $(F=5,96 ; F=0.89$, and $p=0.496)$. The iliocostalis muscle analysis (Figure $2 b$ ) showed no statistical significant differences between 
exercises $(F=5,96 ; F=1.24$, and $p=0.296)$. Small effect sizes were found for both statistical analyses $(E S<0.1)$.

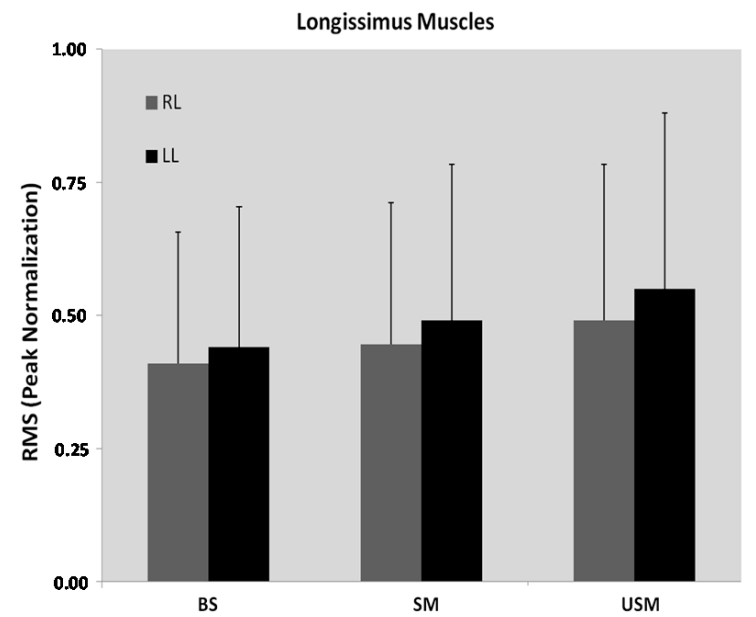

(a)

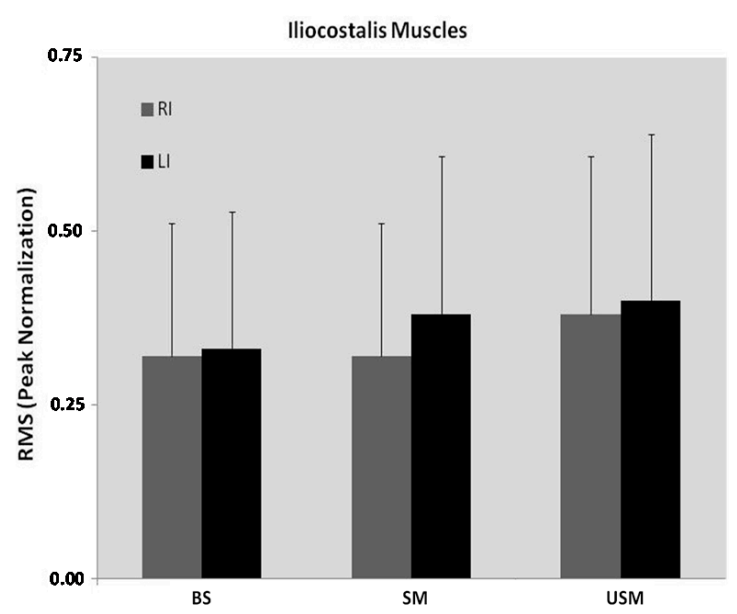

(b)

Figure 2. Grey and black bars are respectively right and left side of muscles: (a) longissimus activity during the bodyweight squat (BS), superman exercises (SM) and unstable superman exercise (USM); (b) iliocostalis activity during the bodyweight squat (BS), superman exercises (SM) and unstable superman exercise (USM).

\section{Discussion}

The primary purpose of our investigation was to evaluate bilateral paraspinal muscle (iliocostalis and longissimus) activity in three traditional core exercises: the superman exercise, the unstable superman exercise and the bodyweight squat. Our results showed a strong correlation between both sides of the longissimus and iliocostalis muscles in the protocol proposed. Additionally, our findings demonstrated that well-trained individuals with no history of low back pain can achieve bilateral muscle recruitment intensity from each of the three exercises assessed.

Our findings are supported by the literature; for example, Callaghan et al. [10] showed similar patterns for each side of the lumbar erector spine and lumbar multifidus muscles during superman exercises, and Oliver et al. [3] observed no significant differences between right and left lumbar multifidus and external oblique muscle activation during superman exercises and flying squirrel core movements.

The second purpose of this investigation was to examine potential muscle activation differences between bodyweight squats and superman exercises (performed on a stable or unstable surface). We did not find significant differences, despite marginally greater mean muscle activation with the unstable superman exercise; it was sufficiently marginal as to not lead to a greater pattern change in all participants. This result does not corroborate our hypothesis, given that we expected an increase of paraspinal muscle recruitment on unstable surface conditions, and maybe there were no differences because we adopted the same range of motion and fixed foot pattern for both superman exercises.

Our results contrast those of previous studies that found differences between stable and unstable surfaces [8,9]. Body position plays a major role in muscle activation, dislocating the body center of mass, creating a more unstable environment; for example, Sternlicht et al. [11] found almost $50 \%$ more muscle activity in upper and lower rectus abdominis muscles and external obliques when abdominal crunches were performed with an instability ball positioned at the lower back compared to when the ball was positioned between scapulae. In order to avoid the potential effects of postural positioning differences on muscle recruitment and focus on effects derived from the unstable surface, properly, as presented in Sternlicht et al. [11], our study analyzed the superman exercise position permitting only the same 
range of motion for both stable and unstable conditions, with the feet fixed. These specifications allowed us to assess the effects of an unstable surface specifically, and it demonstrated that the stability does not produce any variation in trunk muscle recruitment in well-trained individuals.

In relation to bodyweight squats and variations of superman exercises, our results also did not find a difference in trunk muscle recruitment. This may be related to the executional characteristics of the squat: In the descendent phase, forward trunk flexion is restricted by active contraction of paraspinal muscles, which stabilizes the vertebral spine and reduces compression load and shear stress on soft tissues. Squats maintain muscle contraction for a great part of the movement cycle [21-23], in contrast to the relaxation-contraction cycles that characterize superman exercises.

The front barbell squat with a fixed load and the unstable superman exercise both elicited erector spinae muscles equally [14]. Our results corroborated previous findings by demonstrating no differences between the bodyweight squat and superman exercise, regardless of whether it is performed on a stable or unstable surface. Hamlyn et al. [12] demonstrated, via EMG, no augmentation of upper lumbar erector spinae and lumbosacral erector spinae muscles compared to superman exercises, barbell squats and barbell deadlifts with a fixed load. Therefore, it appears that traditional hyperextension exercises (stable and unstable superman exercises) are not superior to multi-joint squats and deadlifts. Furthermore, multi-joint exercises such as the bodyweight squat are more related to functional daily activities or sports "play positions" than are isolation exercises. In accordance with our study, Gottschall et al. [24] reported more activation of lumbar erector spinae and thoracic erector spinae muscles in exercises that also stimulate the shoulder and hip complexes than superman exercises stimulate erector spinae muscles.

To our knowledge, this is the first study comparing EMGs of longissimus and iliocostalis muscles during a bodyweight squat and during superman exercises without the use of accessories. There is an increase in paraspinal muscle recruitment with progressive loads [13] and fixed loads [12,14], which may affect the kinematics of movement. McKean et al. [15] found differences in the kinematics of an unloaded squat versus a 50\% bodyweight load in the initial and final angles of the lumbar and sacrum during the movement. Therefore, it seems reasonable for future researchers to analyze bodyweight movements without devices and to investigate these parameters in women and individuals with low back pain.

Although the aim of some rehabilitation programs is to decrease low back pain by strengthening lumbar extensor muscles, exercises with higher shear and compression forces in the vertebral discs may result in undesired outcomes. Superman exercises exert approximately $4500 \mathrm{~N}$ of load compression between the L4 and L5 disc [10]; another investigation reported approximately $2000 \mathrm{~N}$ of load compression between L4 and L5 in a bodyweight squat [25], which led to the argument that superman exercises can produce twice as much spine compression as can a bodyweight squat. In the present study, a bodyweight squat elicited the same level of iliocostalis and longissimus activity as did traditional superman exercises on stable and unstable surfaces. This indicates that squats may be a more appropriate exercise for paraspinal muscles in the early stages of low back pain rehabilitation, because they produce similar EMG activity on paraspinal muscles along with lower compression loads on the vertebral spine.

Future research must also investigate and compare deep paraspinal and abdominal wall muscle activity analyses on these exercises as well as on the lumbar multifidus's control of spine orientation and intersegmental motion [26], the transversus abdominis's role on posture anticipatory adjustments [27], and the internal and external obliques' effect on pelvis stabilization during multiple tasks [28]. In addition, deep paraspinal and abdominal wall muscles, which have a major role in spine stabilization, were not tested via surface electromyography in the present study.

Finally, the possible limitations of EMG used alone should be noted, so future findings should also provide kinematic and kinetic information regarding these exercises. Another important limitation was the fact that all participants in this study were healthy, well-trained men with no history of low back pain and who had been injury-free for the one-year period leading up to the study. Future investigations 
must involve individuals who are suffering with low back pain in order to verify potential changes in muscle patterns.

\title{
5. Conclusions
}

The current study showed that the bodyweight squat exercise produces muscle activity similar to that of the posterior trunk extensors and iliocostalis and longissimus muscles in superman exercises and unstable superman exercises. Therefore, bodyweight squats seem to be a more reasonable choice in strength programs targeting paraspinal muscles, because there is less risk of compressive load to the spine which may have detrimental effects. This finding also allows us to suppose that the bodyweight squat may be a better choice than superman exercises for lower-back rehabilitation. However, this still must be investigated with a larger sample of men and women of varying ages-with and without low back pain—to determine the bodyweight squat's specific rehabilitation applicability.

Author Contributions: Fernando Carvalheiro Reiser, Bruno Gonçalves Durante and William Cordeiro de Souza conducted the acquisition of data; Fernando Carvalheiro Reiser, Bruno Gonçalves Durante, William Cordeiro de Souza, Luis Paulo Gomes Mascarenhas and Giordano Márcio Gatinho Bonuzzi wrote the main paper. All authors discussed the study conception and design, the results and implications at all stages.

Conflicts of Interest: The authors declare no conflict of interest.

\author{
Abbreviations \\ BS Bodyweight Squat \\ SM Superman Exercise \\ USM Unstable Superman Exercise
}

\section{References}

1. Leetun, D.T.; Ireland, M.L.; Willson, J.D.; Ballantyne, B.T.; Davis, I.M. Core stability measures as risk factors for lower extremity injury in athletes. Med. Sci. Sports Exerc. 2004, 36, 926-934. [CrossRef] [PubMed]

2. McGill, S. Core training: Evidence translating to better performance and injury prevention. Strength Cond. J. 2010, 32, 33-46. [CrossRef]

3. Oliver, G.D.; Stone, A.J.; Plummer, H. Electromyographic examination of selected muscle activation during isometric core exercises. Clin. J. Sport Med. 2010, 20, 452-457. [CrossRef] [PubMed]

4. Hultman, G.; Nordin, M.; Saraste, H.; Ohlesen, H. Body composition, endurance, strength, cross sectional area and density of MM erector spine in men with and without low back pain. J. Spinal Disord. 1993, 6, 114-123. [CrossRef] [PubMed]

5. McGill, S.M.; Hughson, R.L.; Parks, K. Changes in lumbar lordosis modify the role of the extensor muscles. Clin. Biomech. 2000, 15, 777-780. [CrossRef]

6. Luoto, S.; Heliövaara, M.; Hurri, H.; Alaranta, H. Static back endurance and the risk of low-back pain. Clin. Biomech. 1995, 10, 323-324. [CrossRef]

7. Hides, J.A.; Stanton, W.R.; McMahon, S.; Sims, K.; Richardson, C.A. Effect of stabilization training on multifidus muscle cross-sectional area among young elite cricketers with low back pain. J. Orthop. Sports Phys. Ther. 2008, 38, 101-108. [CrossRef] [PubMed]

8. Behm, D.G.; Leonard, A.M.; Young, W.B.; Bonsey, W.A.C.; MacKinnon, S.N. Trunk muscle electromyographic activity with unstable and unilateral exercises. J. Strength Cond. Res. 2005, 19, 193-201. [CrossRef] [PubMed]

9. Ekstrom, R.A.; Osborn, R.W.; Hauer, P.L. Surface electromyographic analysis of the low back muscles during rehabilitation exercises. J. Orthop. Sports Phys. Ther. 2008, 38, 736-745. [CrossRef] [PubMed]

10. Callaghan, J.P.; Gunning, J.L.; McGill, S.M. The relationship between lumbar spine load and muscle activity during extensor exercises. Phys. Ther. 1998, 78, 8-18. [CrossRef] [PubMed]

11. Sternlicht, E.; Rugg, S.; Fujii, L.L.; Tomomitsu, K.F.; Seki, M.M. Electromyographic comparison of a stability ball crunch with a traditional crunch. J. Strength Cond. Res. 2007, 21, 506-509. [CrossRef] [PubMed]

12. Hamlyn, N.; Behm, D.G.; Young, W.B. Trunk muscle activation during dynamic weight-training exercises and isometric instability activities. J. Strength Cond. Res. 2007, 21, 1108-1112. [CrossRef] [PubMed] 
13. Nuzzo, J.L.; McCaulley, G.O.; Cormie, P.; Cavill, M.J.; McBride, J.M. Trunk muscle activity during stability ball and free weight exercises. J. Strength Cond. Res. 2008, 22, 95-102. [CrossRef] [PubMed]

14. Comfort, P.; Pearson, S.J.; Mather, D. An electromyographical comparison of trunk muscle activity during isometric trunk and dynamic strengthening exercises. J. Strength Cond. Res. 2011, 25, 149-154. [CrossRef] [PubMed]

15. McKean, M.R.; Dunn, P.K.; Burkett, B.J. The lumbar and sacrum movement pattern during the back squat exercise. J. Strength Cond. Res. 2010, 24, 2731-2741. [CrossRef] [PubMed]

16. Faul, F.; Erdfelder, E.; Lang, A.G.; Buchner, A. G*Power 3: A flexible statistical power analysis program for the social, behavioral, and biomedical sciences. Behav. Res. Met. 2007, 39, 175-191. [CrossRef]

17. Hermens, H.J.; Freriks, B.; Disselhorst-Klug, C.; Rau, G. Development of recommendations for SEMG sensors and sensor placement procedures. J. Electromyogr. Kinesiol. 2000, 10, 361-374. [CrossRef]

18. Reiser, F.C.; de Moura, J.A.R.; Cardoso, J.M.D.; Grzelczak, M.T.; de Souza, W.C.; Mascarenhas, L.P.G. Eletromiografia do exercício de crucifixo em diferentes planos e angulações de movimento. Rev. Bra. Prescr. Fisiol. Exer. 2014, 8, 864-870. (In Portuguese)

19. De Luca, C.J. The use of surface electromyography in biomechanics. J Appl. Biomech. 1997, 13, $135-163$. [CrossRef]

20. Mannion, A.F.; Connolly, B.; Wood, K.; Dolan, P. The use of surface EMG power spectral analysis in the evaluation of back muscle function. J. Rehabil. Res. Dev. 1997, 34, 427.

21. Hartmann, H.; Wirth, K.; Klusemann, M. Analysis of the load on the knee joint and vertebral column with changes in squatting depth and weight load. Sports Med. 2013, 43, 993-1008. [CrossRef] [PubMed]

22. Hartmann, H.; Wirth, K.; Mickel, C.; Keiner, M.; Sander, A.; Yaghobi, D. Stress for vertebral bodies and intervertebral discs with respect to squatting depth. J. Funct. Morphol. Kinesiol. 2016, 1, 254-268. [CrossRef]

23. Reiser, F.C.; de Souza, W.C.; Mascarenhas, L.P.G. Cinética e Cinemática do Agachamento na Coluna Vertebral: Estudo de Revisão. Rev. Unian. 2015, 16, 7-13. (In Portuguese) [CrossRef]

24. Gottschall, J.S.; Mills, J.; Hastings, B. Integration core exercises elicit greater muscle activation than isolation exercises. J. Strength Cond. Res. 2013, 27, 590-596. [CrossRef] [PubMed]

25. Bazrgari, B.; Shirazi-Adl, A.; Arjmand, N. Analysis of squat and stoop dynamic liftings: Muscle forces and internal spinal loads. Eur. Spine J. 2007, 16, 687-699. [CrossRef] [PubMed]

26. Moseley, G.L.; Hodges, P.W.; Gandevia, S.C. Deep and superficial fibers of the lumbar multifidus muscle are differentially active during voluntary arm movements. Spine 2002, 27, E29-E36. [CrossRef] [PubMed]

27. Hodges, P.W.; Richardson, C.A. Transversus abdominis and the superficial abdominal muscles are controlled independently in a postural task. Neurosci. Lett. 1999, 265, 91-94. [CrossRef]

28. Badiuk, B.W.; Andersen, J.T.; McGill, S.M. Exercises to activate the deeper abdominal wall muscles: The lewit: A preliminary study. J. Strength Cond. Res. 2014, 28, 856-860. [CrossRef] [PubMed] 